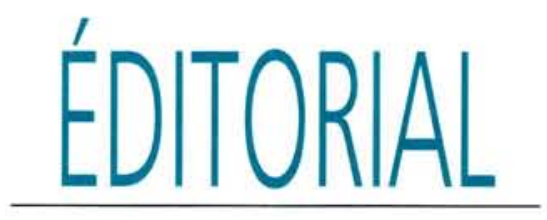

JEAN-PAUL MOATTI

\title{
La lutte contre le Sida : à la recherche d'un second souffle
}

$\mathrm{L}$ a dixième Conférence Internationale annuelle sur le Sida s'est tenue à Yokohama au mois d'août dernier. Comme les précédentes, elle se voulait un moment de la prise de conscience planétaire de la globalisation de l'épidémie avec cette fois une insistance particulière sur la situation en Asie où vit plus de la moitié de la population mondiale, et où la situation est très préoccupante. A partir de 1995, c'est en Asie que devrait survenir le nombre le plus élevé de nouvelles contaminations.

En réalité, la réunion de Yokohama a essentiellement exprimé une "banalisation" de la lutte contre le Sida ; c'est ainsi que la Conférence ne se tiendra plus désormais que tous les deux ans. Cette banalisation est, dans une certaine mesure, le produit des progrès accomplis (avancées thérapeutiques, notamment en matière d'infections opportunistes) et d'un plus grand réalisme face aux difficultés rencontrées (concernant, tout particulièrement, la mise au point d'un vaccin préventif à court terme). II faut maintenant s'installer dans la durée : il est nécessaire d'améliorer la connaissance fondamentale des mécanismes moléculaires et cellulaires du vivant ; par ailleurs, pas d'avancée majeure à attendre en matière de prévention sans compréhension plus approfondie des déterminants sociaux des comportements sexuels ou toxicomanes.
Malheureusement, le sentiment de banalisation ressenti par de nombreux participants de la conférence de Yokohama s'accompagne de légitimes inquiétudes quant à une possible remise en cause des principes fondamentaux qui ont jusqu'à présent présidé à la mobilisation mondiale contre le Sida et que ces Congrès "pas comme les autres" (où chercheurs et médecins côtoyaient les malades eux-mêmes) avaient permis d'incarner. Pour Jonathan Mann, ancien Directeur du Programme OMS, le Sida a été l'occasion d'une « rencontre imprévue et révolutionnaire entre la santé publique et les droits de l'homme". Or, il est significatif que la Conférence Mondiale sur le Sida de Boston ait été annulée il y a deux ans (et transférée à Amsterdam) pour protester devant le refus des autorités américaines d'amender la législation en vigueur qui permet d'interdire l'entrée de leur territoire à une personne séropositive, et que pas une seule voix ne se soit élevée sur ce sujet à propos de Yokohama, alors que la législation japonaise en cette matière est plus répressive encore. En France même, le consensus qui s'était établi jusqu'à présent parmi les experts et les élites constituées (à la notable exception de l'extrême-droite lepéniste) autour du refus de toute tentation coercitive dans les politiques publiques de lutte contre l'épidémie tend à s'éroder doucement avec les prises de position en faveur d'un dépistage obligatoire ou de la levée du secret médical dans diverses circonstances, toutes mesures dont l'inefficacité en santé publique semble bien établie, mais qui ne sont pas forcément sans efficacité idéologique auprès de l'opinion.

En définitive, l'avenir de la lutte contre le Sida se joue peut-être entre ces deux formes opposées de banalisation. Celle qui, à la faveur de la concentration progressive de la maladie dans les pays et les groupes les plus défavorisés, sacrifierait plus ou moins insidieusement les droits des personnes et communautés atteintes à la "nécessaire" protection de la collectivité. Celle au contraire qui continuerait plus que jamais à faire de la confrontation au Sida un révélateur des limites et des conservatismes de nos pratiques médicales, de nos institutions et formes de solidarité sociale, voire de notre citoyenneté démocratique. C'est dans cette seconde perspective que Natures, Sciences, Sociétés ne manquera pas d'aborder dans les mois qui viennent, de façon plus approfondie, les multiples enjeux scientifiques, sociétaux et éthiques que soulève cette maladie. 